\title{
Adult attention-deficit hyperactivity disorder: recognition and treatment in general adult
}

\section{psychiatry}

\section{PHILIP ASHERSON, WAI CHEN, BRIDGET CRADDOCK and ERIC TAYLOR}

\author{
Summary Attention-deficit hyper- \\ activity disorder (ADHD) is a common \\ disorder affecting children and adults. \\ Many young people treated with \\ stimulants, as well as those in whom \\ ADHD went unrecognised in childhood, \\ need treatment as adults. Stimulants and \\ atomoxetine effectively reduce ADHD \\ symptoms at all ages and should be a \\ standard treatment in general adult \\ psychiatric practice.
}

\section{Declaration of interest The authors research is funded by the UK Medical \\ Research Council, the WellcomeTrust and the US National Institute of Mental Health. P.A. received unrestricted research funds from Janssen-Cilag and has spoken at meetings sponsored by Janssen-Cilag and Eli Lilly.}

Most child and adolescent mental health services recognise the existence - and need for treatment - of a condition characterised by high levels of inattentiveness, impulsiveness and restless overactivity. The symptoms defining attention-deficit hyperactivity disorder (ADHD) are regarded as a source of disability in children and adolescents and a risk to adult psychological adjustment. Numerous specialist multidisciplinary ADHD clinics have been developed and many paediatricians include treatment of ADHD as an important part of their clinical activity.

A good deal of the justification for this therapeutic activity is the demonstration that ADHD is a predictor of adult mental health problems. Furthermore, effective treatment with stimulant medication increases the immediate value of the clinical diagnosis (Taylor et al, 2004). General adult psychiatry, however, has not yet followed suit in identifying and treating substantial numbers of affected people. Yet it is likely that an increasing load in adult psychiatry will develop. Young people are entering adult life while still receiving medication for ADHD, and adult psychiatrists are needed to take over psychiatric treatment when symptoms persist. An increasing number of adults are recognising themselves - or being recognised by family members, general practitioners, probation officers, drug and alcohol specialists, psychologists and others - as being disabled by ADHD and requiring assistance. In addition there is a pool of adult psychiatric patients in whom the diagnosis of ADHD has gone unrecognised and for whom treatments put in place for alternative diagnoses, such as anxiety, depression, cyclothymia and personality disorder, are ineffective.

\section{VALIDITY OF THE ADULT DIAGNOSIS}

Evidence for the validity of adult ADHD and descriptions of its clinical diagnosis and management are given in detail elsewhere (Faraone et al, 2000; Weiss et al, 2002; Weiss \& Murray, 2003; Asherson, 2005). The disorder in adults is not as well studied as it is in children. However, there are sufficient data and unanimous agreement among clinical experts on the validity of the adult diagnosis. Although there is evidence that ADHD symptoms show an age-dependent decline, there is no evidence that the disorder disappears in adolescence and adulthood, and considerable evidence that in the majority symptoms persist and are frequently associated with clinical and psychosocial impairments.

Furthermore, it is well established that ADHD symptoms in adults show the same responsiveness to stimulant and non-stimulant medications as that seen in children (Faraone et al, 2004; Simpson \& Plosker, 2004). For these reasons UK and European experts in a recent review of available data on the efficacy of extended-release preparations in the treatment of ADHD include recommendations for their use in adults (Banaschewski et al, 2006).

A key question is the number of children with ADHD who continue to show ADHD in adult life. Studies that followed children identified with ADHD, as well as controls, into adulthood have been included in a meta-analysis of 32 publications (Faraone et al, 2006). When the ADHD samples included only those who met full diagnostic criteria for ADHD the rate of persistence was approximately $15 \%$ at age 25 years. However, the rate was far higher - approximately $65 \%$ - when individuals fulfilling the DSM-IV definition of ADHD in partial remission were included, referring to the persistence of some symptoms associated with significant clinical impairments.

Our own data support this conclusion. In an epidemiological survey of schoolchildren from the London district of Newham, E.T. and colleagues measured ADHD behaviours at ages 7 and 17 years. Levels of inattentive and restless activity had indeed diminished. However, 17-yearolds previously characterised as 'hyperactive' showed a level of hyperactivity similar to that seen in the control group of normal 7-year-olds. Although this meant that most no longer met full diagnostic criteria, the relative distance between them and controls was unchanged. Importantly, the degree to which hyperactive behaviour was above the age norm was strongly related to poor social adjustment in terms of lack of friends, occupation and constructive activity. Deficits associated with high levels of psychiatric morbidity were also seen when the same people were followed up at age 26 years (further information available on request) and these are all the more significant because of increasing demands in adult life for selforganisation and the ability to plan ahead.

\section{DIAGNOSIS IN ADULTS}

Wender (1998) provided a list of adult behaviours linked to childhood ADHD (see also Weiss \& Murray, 2003; Asherson, 2005). Motor hyperactivity may be replaced by a subjective sense of restlessness, difficulty in relaxing and settling down and dysphoria when inactive. Attention deficits may well persist in a lack of concentration on detail, the need to re-read materials several times, forgetting activities and appointments, losing things and losing the thread of conversations. Thoughts are unfocused and 'on the go' all the time. Mood changes are often rapid shifts into depression or excitability, 
irritability and temper outbursts that interfere with personal relationships. Disorganisation is prominent, tasks are not completed, problem-solving is lacking in strategy and time management is particularly poor. Impulsivity continues and leads to problems in teamwork, abrupt initiation and termination of relationships, and a tendency to make rapid and facile decisions without full analysis of the situation.

Although most people experience such symptoms at times, individuals with ADHD experience these to a severe degree most of the time. As in other common adult psychiatric disorders, such as anxiety and depression, ADHD symptoms are continuously distributed throughout the population (Asherson, 2004). Clinical thresholds are therefore determined on the basis of usual clinical practice, which recognises disorder when the symptoms are sufficiently frequent or severe to cause personal distress, distress to others, interference with psychosocial function or health complications.

In making the correct diagnosis there are potential traps for the inexperienced ADHD diagnostician. First, ADHD symptoms may be not be evident in the clinic or other highly structured environments. The reason is that ADHD symptoms may respond to (decrease in) situations that are highly novel or salient to the individual, such as an important psychiatric evaluation. It is therefore important to base mental state descriptions on a typical week and a variety of normal situations. Second, ADHD symptoms start early in life, by definition before the age of 7 years, and are persistent and non-episodic. They are therefore more trait-like than symptom-like, since there is no clear change from a premorbid state, and can therefore be mistaken for character traits that may be deemed resistant to psychopharmacological intervention. Third, mood instability is extremely common in adult ADHD and can lead to diagnoses of minor affective disorders or personality disorder. Clinical experience suggests that mood instability in the context of ADHD frequently responds to stimulants in the same timescale as 'core' symptoms of the disorder.

Many adults with ADHD have other problems: antisocial personality, alcohol misuse, substance dependence, dysthymia, cyclothymia, anxiety disorders and general and specific learning difficulties. Early-onset and persistent antisocial behaviour is a particularly common association that sometimes leads to persistent impulsive antisocial

PHILIPASHERSON, MRCPsych, PhD, WAI CHEN, MRCPsych, MRC Social, Genetic and Developmental Psychiatry Centre, Institute of Psychiatry, London; BRIDGET CRADDOCK, BSc, MB, ChB, MRCPsych, Glyncynffig Hostel, Bridgend; ERIC TAYLOR, FRCPsych, FRCP, PhD, MRC Social, Genetic and Developmental Psychiatry Centre, Institute of Psychiatry, London, UK

Correspondence: Philip Asherson, MRC Social, Genetic and Developmental Psychiatry Centre, Institute of Psychiatry, Denmark Hill, London SE5 8AF, UK. Email: p.Asherson@iop.kcl.ac.uk

(First received 15 May 2006, accepted 18 August 2006)

behaviour in adult life. Longitudinal studies show that ADHD comes first and may then lead to development of antisocial behaviour, a trajectory thought to be mediated by prominent shared environmental influences.

Bipolar affective disorder includes some symptoms that are very similar to those of ADHD. Both are characterised by overactivity, distractibility, inattentiveness and mood changes. The definitions were not framed with the purpose of distinguishing between these two disorders; however, the distinction is usually not difficult. The mood state of ADHD is irritable and volatile, rather than containing elements of euphoria and grandiosity. The course of adult ADHD is one of a persisting trait, very different from the episodes of mania and/or depression characteristic of bipolar disorder. Follow-up studies of typical ADHD cases have not observed the development of classic adult bipolar disorder (Type 1). Recent changes have taken place in the concept of 'juvenile mania'. It has been argued that mania of very early onset is characterised by a mood of irritability rather than euphoria, and by chronicity rather than fluctuation. If this change of definition is accepted, then the distinction from ADHD becomes difficult indeed. In people with a pattern of ADHD symptoms, emotional instability and irritability in childhood, the mood symptoms tend to persist in the same form rather than becoming more like classic bipolar disorder.

\section{CLINICAL IMPLICATIONS}

Childhood ADHD often persists into adulthood, either as a categorical diagnosis or as persistence of symptoms which contribute to poor social adjustment, personality problems and psychiatric comorbidity. Many young people currently treated with stimulants will continue this medication into adult life, requiring psychiatric advice. Many children with ADHD go unrecognised and may present in adulthood for the first time. Mis-specification of the diagnosis currently leads to inappropriate, ineffective and protracted interventions by psychiatrists and general practitioners. Furthermore, adults with untreated ADHD use more healthcare resources because of smoking-related disorders, increased rates of serious accidents and alcohol and drug misuse. Further research is now required to quantify accurately the contribution of ADHD to adult psychopathology and thus to psychiatric morbidity. Medication, especially with stimulant drugs, is an effective means of reducing ADHD symptoms and behaviours in adulthood. For these reasons we strongly urge that appropriate drug treatment of ADHD should be a normal part of the therapeutic resources available within general adult psychiatry.

\section{REFERENCES}

Asherson, P. (2004) Attention-deficit hyperactivity disorder in the post-genomic era. European Child and Adolescent Psychiatry, I3 (suppl. I), i50-i70.

Asherson, P. (2005) Clinical assessment and treatment of attention deficit hyperactivity disorder in adults. Expert Review of Neurotherapeutics, 5, 525-539.

Banaschewski, T., Coghill, D., Santosh, P., et al (2006) Long-acting medications for the hyperkinetic disorders: a systematic review and European treatment guideline. European Child and Adolescent Psychiatry, in press.

Faraone, S.V., Biederman, J., Spencer, T., et al (2000) Attention-deficit/hyperactivity disorder in adults: an overview. Biological Psychiatry, 48, 9-20.

Faraone, S. V., Spencer, T., Aleardi, M., et al (2004) Meta-analysis of the efficacy of methylphenidate for treating adult attention-deficit/hyperactivity disorder. Journal of Clinical Psychopharmacology, 24, 24-29.

Faraone, S. V., Biederman, J. \& Mick, E. (2006) The age-dependent decline of attention deficit hyperactivity disorder: a meta-analysis of follow-up studies. Psychological Medicine, 36, 159-165.

Simpson, D. \& Plosker, G. L. (2004) Atomoxetine: review of its use in adults with attention deficit hyperactivity disorder. Drugs, 64, 205-222.

Taylor, E., Dopfner, M., Sergeant, J., et al (2004)

European clinical guidelines for hyperkinetic disorder first upgrade. European Child and Adolescent Psychiatry, 13 (suppl. I), i7-i30.

Weiss, M. \& Murray, C. (2003) Assessment and management of attention-deficit hyperactivity disorder in adults. Canadian Medical Association Journal, 168, 715-722

Weiss, M., Murray, C. \& Weiss, G. (2002) Adults with attention-deficit/hyperactivity disorder: current concepts. Journal of Psychiatric Practice, 8, 99-III.

Wender, P. H. (1998) Attention-deficit hyperactivity disorder in adults. Psychiatric Clinics of North America, 21 $761-774$. 